\title{
Significant Reduction in Gender Grade Disparities in a Reformed Introductory Physics Course
}

\author{
Wendell H. Potter, Charles J. De Leone, Catherine M. Ishikawa, \\ Jacob A. Blickenstaff, and Patrick L. Hession \\ University of California, Davis
}

\begin{abstract}
In 1996 we switched from a traditionally structured introductory physics course for biological science majors at the University of California, Davis to a reformed course incorporating the implications for instruction that follow from a constructivist view of learning. In the traditional course there was a persistent and stable pattern of course letter-grade distributions in which males received a disproportionate share of high grades and females received a disproportionate share of low grades. This gender disparity has been significantly reduced for the same population of students in the reformed course. This paper is a preliminary report of this data.
\end{abstract}

\section{The Reformed Course}

In the fall quarter of 1996, we implemented an active-learning based reformed introductory physics course ${ }^{1}$, Physics 7, which is taken by $\sim 1200$ biological science majors each year at the University of California, Davis. Physics 7 replaced the traditionally taught lecture/lab course, Physics 5, that had been offered essentially unchanged for several decades. We have attempted to incorporate the implications for instruction arising from a constructivist understanding of learning to as great a degree as possible in Physics 7, subject only to the limits imposed by a few fixed constraints (no increase in classroom space, no increase in instructional resources, and course to remain a 4-unit year-long sequence to accommodate $\sim 1200$ students per year).

Significant changes were made in content, in the mode and format of instruction, in assessment of student learning, and in the roles of students and instructors.

- Content is organized around a small number of widely applicable models, rather than being a sequential presentation of a large number of topics.
The textbook has been replaced with a relatively small set of class notes.

- Instruction is centered in the 2.5 hour discussion/lab section, which meets twice weekly (5 hours/week). The 25 students in each discussion/lab section work collaboratively in groups of five. Homework assigned after each discussion/lab meeting is an extension of the activities just completed. The discussion/lab is supplemented with one 80 minute weekly lecture.

- Approximately a half hour of each weekly lecture is devoted to quizzes, which always involve verbal explanation and analysis (and additionally sometimes require numerical calculations). Quizzes, as well as the final, are graded using rubrics based on categories of expected performance. Grades are not curved.

- The student learning goals for the activities in the discussion/lab and for the homework assignments focus on "making sense" of physical phenomena by developing a coherent understanding in terms of prior understandings (often modified) and new models. The instructor's role in the discussion/lab section is to facilitate students' active construction of understanding. Graduate 
teaching assistants constitute the majority of discussion/lab instructors.

The Physics 7 course has been incrementally evolving since 1996 in response to formative course assessments embedded in course activities.

\section{Observed Differences in Grade Distributions by Gender}

Figure 1 shows normalized grade histograms separated by gender for (a) the traditionally taught Physics 5A course the last time it was taught (winter quarter 1996) and (b) the first full offering of the reformed Physics 7A course (fall quarter 1996).

\section{Normalized Grade Histograms}

(a) Physics 5A Winter 96

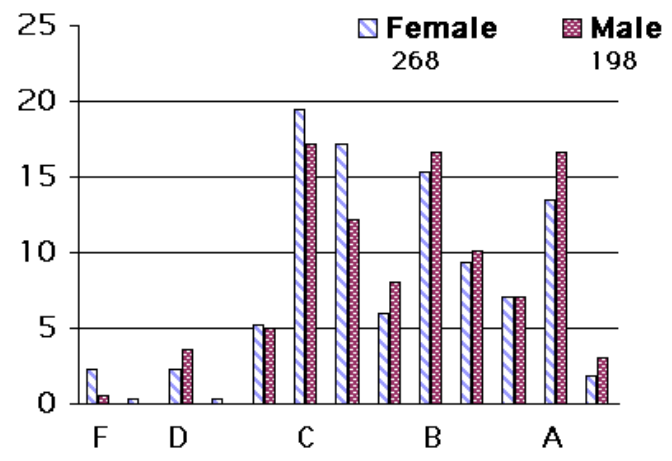

\section{(b) Physics 7A Fall 96}

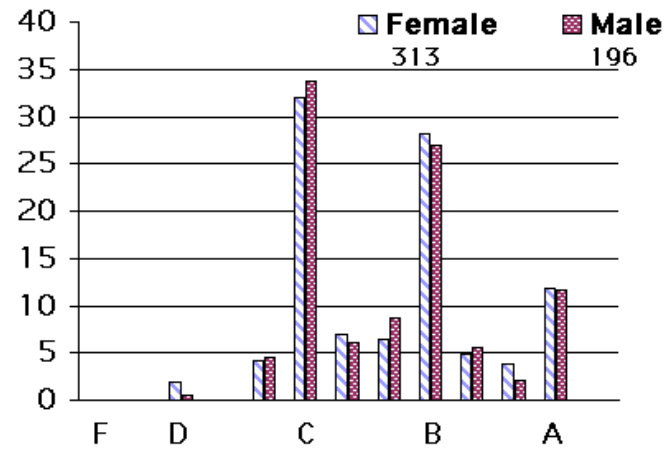

Figure 1. The grade histograms in (a) are from the last offering of the traditionally taught Physics 5 course at UC Davis. The histograms in (b) are from the first offering of Physics 7, the reformed course that replaced Physics 5.

A disparity can be seen in the Physics 5A data in the percentage of females in the
" $\mathrm{C}+$ " range and below compared to males, which is not present in the Physics 7A data. The gender disparity seen in this particular quarter appears in all Physics 5 quarters we have examined. On the other hand, in Physics 7, the gender disparity is either absent, as in the fall 1996 data shown in Fig. 1b, or is significantly smaller than in the Physics 5 course.

It is difficult to make direct visual comparisons among different courses or between genders when using binned data, especially when the means and modes of the distributions are different. Quantitative comparisons are also difficult, since, in general, relative heights of adjacent bins are a function of the location of bin edges with respect to features of the distribution, such as location of the mean. Figure 2 displays the same information represented in Figure 1 as grade-probability densities.

\section{Grade Probability Densities}

(a) Physics 5A Winter 96
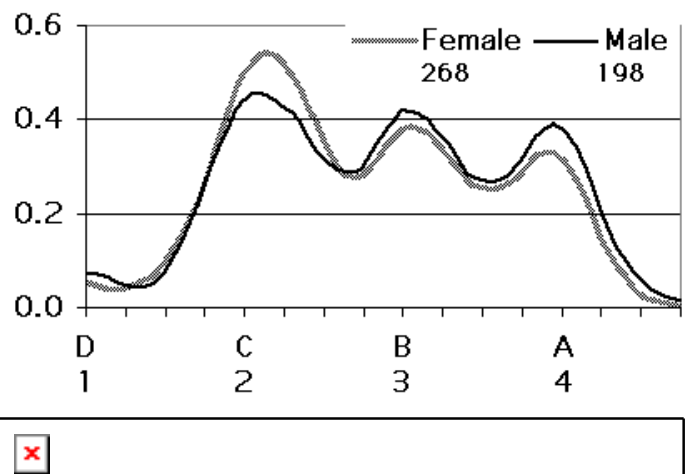

Figure 2. Grade probability densities, generated from the originally reported letter grades in the courses that generated the histograms shown in Figure 1. 
We generate the smoothed gradeprobability density curves, which facilitate the visual interpretation of information contained in the grade distributions, using a kernel estimator method ${ }^{2}$. This particular smoothing approach preserves the information in the original binned data, e.g., the location of the mean; it also does not introduce new or spurious features not present in the original data. The details of this smoothing method are briefly outlined in the appendix to this paper.

We show in Fig. 3 grade-probability densities for the A-quarters of the last three years of the traditionally taught Physics 5 course and of the two years 1997-1999 for the reformed Physics 7 course.

\section{Grade Probability Densities}

(a) Physics 5A 1994-96

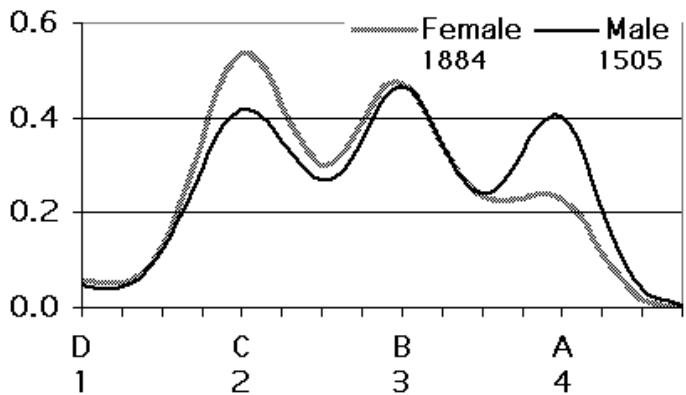

(b) Physics 7A 1997-99

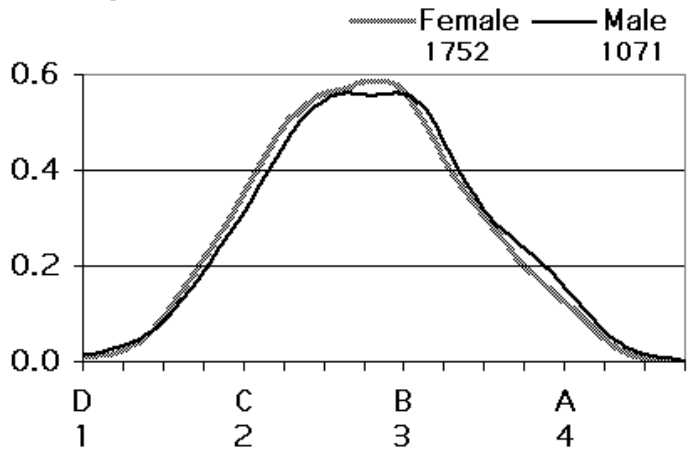

Figure 3. Grade probability density functions for males and females in (a) the traditionally taught Physics 5 (1994-1996) and in (b) the reformed Physics 7 (1997-1999).
Before addressing the clearly evident gender grade disparity in the traditionally taught Physics 5 data, we first discuss the difference in overall shape of the Physics 5 and Physics 7 distributions. This difference in overall shape has to do with differences in the way letter grades are assigned and is totally independent of the gender grade disparity.

The Physics 5 data presented in Fig. 3a is a compilation from the 21 independently graded lecture sections that were offered over the three-year time span. Most instructors used the same method to map a numerical course grade to a letter grade. The method consisted of establishing the "break points" between the letter grades based on several criteria, one of which was often the shape of the numerical distribution itself. Then, a small number of grades near the break points were adjusted up or down by affixing a "+" or "-" to the letter grade. The resulting smaller number of "in-betweengrades" is the reason for the dips in the smoothed grade-probability density curves.

In the first couple of offerings of Physics 7, we followed the same basic mapping procedure. The "dips" in the 1996 Physics 7 data shown in Fig. 2b are even deeper than in the Physics 5 data. However, beginning in fall 1997, we began making absolute grade point assignments for each piece of graded work. The final course grade is then simply an average of 30 to 50 independently assigned grade points, which automatically leads to a smooth distribution without "humps."

The disparity in the distribution of low and high grades by gender displayed in the Physics 5 data in Fig. 3 a has been observed in every lecture section we have examined. In some sections it is somewhat more pronounced and in others less pronounced. The Physics 5 data shown in Fig. 2a represents one of the quarters when the disparity is less pronounced. We are just in 
the beginning phase of trying to sort out dependencies of this disparity on instructor lecture style and on other course features.

The two year accumulated data from Physics 7 displayed in Fig. 3b show only a very small difference in grade distribution by gender. There are no known differences in the populations of students who took Physics 5 and Physics 7 between 1994 and 1999. Students in essentially all biological science majors at UC Davis are required to take this physics sequence, and very few students from other majors take it (there are two other introductory physics sequences, one at a lower and one at a higher level than Physics 5/7). Consequently, there is little possibility that self selection of courses by students caused any change in the populations of students taking the two courses. We conclude, therefore, that the nearly complete elimination of the grade disparity by gender in Physics 7 is due solely to course features and not to changes in the populations of students taking the courses.

By looking closely at the small differences that are apparent in some quarters, we hope to be able to isolate those features of the Physics 7 course that are most significant in eliminating the gender grade disparity.

\section{Summary}

Significant differences in grade distributions as a function of gender existed in the traditionally taught introductory physics course for biological science majors at UC Davis. These differences have been essentially eliminated in the reformed Physics 7 course, which incorporates the implications for instruction arising from a constructivist understanding of learning.

\section{Appendix}

Each curve shown in Figures 2 and 3 is a plot of the $100+$ calculated values of the grade-probability density function, $f(\mathrm{x})$, over the grade point range -0.5 to 4.75 at intervals of 0.05 . Letter grades are first converted to numerical values with the letter grade " $F$ " assigned 0 and " $A$ " assigned 4.0. Suffixes of "+" or "-" increase or decrease the point value by 0.33 . An unbinned grade, $X_{\mathrm{i}}$, for each student is then generated by randomizing within an interval representing the difference between letter grades $(0.333$ in this case). Values of $f(\mathrm{x})$ are calculated by summing the kernel $K$ over all $N$ values of $X_{\mathrm{i}}$. The kernel estimator is defined as

$$
f(x)=\frac{1}{N h} \sum_{i=1}^{N} K\left(\frac{x-X_{i}}{h}\right)
$$

A window width or smoothing factor, $h$, determines how many $X_{\mathrm{i}}$ contribute to each point of $f(\mathrm{x})$. In all density curves discussed in this paper, a triangular-shaped kernel was used and the smoothing factor, $h$, was set equal to 0.4 , which works well for bin widths of 0.33 . The advantage of using this method as opposed to other smoothing operations, such as a "running average", for example, is that means and areas are preserved and are not sensitive to choice of $h$ or to the shape (e.g., rectangular, triangular, gaussian) of the kernel used. A triangular shaped kernel (or other shape with a central peak) produces a visually smoother curve than does a rectangular shaped kernel.

\section{References}

${ }^{1}$ Potter, W.H., De Leone, C.J., \& Coleman, L.B., "Radically restructured introductory physics course at a large research university" in Redish, E.F. \& Rigden, J.S. The Changing Role of Physics Departments in Modern Universities, Proceedings of ICUPE, Part One: Presentations, 1997, 829-830.

${ }^{2}$ Tarter, M.E. \& Lock, M.D., Model-Free Curve Estimation, Chapman \& Hall, NY, 1993.

\section{Acknowledgements:}

We gratefully acknowledge the support of an NSF grant DUE-9354528 to develop Physics 7.

We wish to thank Corrie Jo Potter for suggesting the use of kernel estimator methods. 